Pablo Cobo Gálvez ${ }^{1}$

\title{
Génesis y elaboración de la Ley de Promoción de la Autonomía Personal y Atención a las Personas en Situación de Dependencia
}

SUMARIO: I. EL LIBRO BLANCO DE LA DEPENDENCIA.- II. LOS GRANDES TEMAS SOBRE LA CONFIGURACIÓN DEL SISTEMA PARA LA AUTONOMÍA Y ATENCIÓN A LA DEPENDENCIA. A). De COBERTURA UNIVERSAL EN El ÁMbito de la protección pública o en el marco regulador de la Seguridad Social. B). PROTECCIÓN DE ACCESO ABIERTO A TODA PERSONA CON DEPENDENCIA O LIMITADO A determinados niveles de severidad C). Prestaciones de servicios o prestacioNES ECONÓMiCAS. D). FinANCIACiÓN a TRAVÉS DE IMPUESTOS GENERALES, FinALISTAS O COTIZACIONES. E). LA DISTRIBUCIÓN DE LA FINANCIACIÓN DEL SISTEMA ENTRE LAS ADMINISTRACIONES PÚBliCAS. F). Debe HABER COFINANCIACIÓN DE LOS BENEFICIARIOS O LAS PRESTACIONES DEBEN SER DE CARÁCTER GRATUITO. G). GESTIÓN CENTRALIZADA O DESCENTRALIZADA EN LAS CCAA Y LOS MUNICIPIOS. III. NEGOCIACIÓN CON LOS INTERLOCUTORES SOCIALES. IV. APROBACIÓN DEL ANTEPROYECTO Y PROYECTO POR EL GOBIERNO: COMISIÓN DE PRESIDENCIA. V. LA PARTICIPACIÓN DE LAS ORGANIZACIONES DE PERSONAS CON DISCAPACIDAD Y MAYORES. VI. LA TRAMITACIÓN PARLAMENTARIA DE LA LEY.

Si analizamos la memoria que acompaña a la Ley 39/2006, de 14 de diciembre, de Promoción de la Autonomía Personal y Atención a las Personas en Situación de Dependencia descubrimos que son múltiples y de gran

${ }^{1}$ Subdirector General de Planificación, Ordenación y Evaluación del IMSERSO. 
calado las causas que justifican la aprobación de esta ley, pero la fundamental es el incremento en los últimos años de forma muy importante de la demanda de cuidados personales, que seguirá aumentando a un fuerte ritmo durante las próximas décadas, como consecuencia de la combinación de factores de carácter demográfico, médico y social, entre los que podemos citar el envejecimiento de la población, las mayores tasas de supervivencia de las personas afectadas por alteraciones congénitas, enfermedades y accidentes graves.

En las últimas décadas la población mayor española ha experimentado un crecimiento muy considerable. El número de personas mayores de 65 años se ha duplicado en los últimos treinta años del siglo XX, alcanzando hoy cifras por encima de los 7, 4 millones de personas mayores de 65 años.

Este fenómeno del envejecimiento va a continuar en los próximos años a la vez que disminuirá la proporción de personas en edades jóvenes, como muestran la evolución prevista de la estructura de la población española. En este crecimiento es necesario destacar el gran aumento que va a experimentar el colectivo de población de 80 y más años, cuyo ritmo de crecimiento será muy superior al del grupo de 65 . La evolución de la composición interna de la población mayor de 65 años nos muestra cómo en el año 2016 tres de cada diez personas mayores de 65 años superarán, a su vez, los 80 .

Por otra parte, han sido las familias las que han asumido el cuidado de las personas dependientes, a través de lo que ha dado en llamarse "apoyo informal". Este hecho coincide en el tiempo con cambios importantes en el modelo de familia debido a la incorporación progresiva de la mujer al mercado de trabajo, lo que hace que el apoyo informal sea insostenible.

La cobertura de las situaciones de dependencia mediante políticas públicas era una de las propuestas de gobierno del Partido Socialista, recogiendo las recomendaciones de los Organismos Internacionales y específicamente de la Unión Europea. De forma expresa el Presidente del Gobierno adquiere el compromiso, en sede parlamentaria, en junio de 2004 de elaborar una Ley para la protección de las situaciones de dependencia, y de elaborar un Libro Blanco de la Dependencia antes del finalizar el año.

\section{EL LIBRO BLANCO DE LA DEPENDENCIA}

La Secretaría de Estado de Servicios Sociales, Familias y Discapacidad encarga al IMSERSO la elaboración del Libro Blanco sobre la Atención a las personas en situación de dependencia en España.

En este Libro y en la documentación complementaria que la acompaña se analizó la información disponible procedente de investigaciones desarro- 
lladas en España en el área de la dependencia, así como los datos suministrados por las Comunidades Autónomas sobre los recursos disponibles en ese momento para atender a las personas dependientes, además de las propuestas y consideraciones realizadas por las Cortes Generales, Defensor del Pueblo, Agentes Sociales, Comunidad Científica, Organizaciones representativas de las personas mayores y de las personas con discapacidad, Entidades Sociales y Organismos Internacionales.

El Libro Blanco sobre la Dependencia se planteó como objetivos: Realizar un diagnóstico riguroso de la situación de las personas dependientes en España y ofrecer alternativas útiles para llevar a cabo la implantación de un Sistema Nacional de Dependencia.

El Libro Blanco se estructura en 12 capítulos, que se organizan para ofrecer una amplia panorámica sobre aspectos demográficos, características y perfiles de la dependencia, régimen jurídico, apoyo informal, recursos para la atención de personas mayores y menores de sesenta y cinco años, atención sanitaria, coordinación socio-sanitaria, presupuestos y recursos económicos dedicados a la Dependencia por el sector público, criterios y técnicas de valoración, generación de empleo y retornos económicos que supondría el desarrollo de un Sistema de atención a las personas dependientes, estudio comparado sobre diferentes sistemas de protección en los países de nuestro entorno y una serie de consideraciones finales que sirvan para la reflexión, el debate, y en su caso, la toma de decisiones en torno a la atención de las personas dependientes.

Como se señala en su presentación, el Libro ofrece dos clases bien diferenciadas de aportaciones. La primera documenta y evalúa la situación de la dependencia en España y analiza también la de aquellos países que nos pueden servir de referencia, con la intención de aportar informaciones y conocimientos que nos permitan contextualizar el debate. La segunda, resumida en el capítulo final, recoge un conjunto de consideraciones y propuestas sistematizadas, en ocasiones de carácter alternativo con el fin de que pudieran ser objeto de análisis en debate técnico por los expertos y político por parte de los partidos políticos y grupos parlamentarios, así como por los Departamentos ministeriales directamente afectados por las propuestas y de forma especial por los Ministerios de Sanidad y de Economía y Hacienda.

Se trata de una aproximación a materias que necesariamente había de abordar el Sistema de Protección: su fundamentación jurídica, la determinación del concepto de la dependencia protegible y los grados de la misma, los órganos y técnicas de valoración, los requisitos de acceso y la estimación de los posibles beneficiarios, las prestaciones y servicios que debería comprender, las posibles vías de financiación y algunas consideraciones sobre la organización y gestión del Sistema y sobre la red de servicios y los proveedores. 


\section{LOS GRANDES TEMAS SOBRE LA CONFIGURACIÓN DEL SISTEMA PARA LA AUTONOMÍA Y ATENCIÓN A LA DEPENDENCIA}

Los temas que durante la elaboración del Libro Blanco y posteriormente en la fase de preparación del proyecto de ley fueron decisivos para configurar el modelo de protección y sobre los que era necesario decidir desde el punto de vista político y técnico eran los siguientes:

a) De cobertura universal en el ámbito de la protección pública o en el marco regulador de la Seguridad Social.

b) De acceso abierto a toda persona con dependencia o limitado a determinados niveles de severidad y, por lo tanto, con aplicación de baremo.

c) De prestación de servicios o de prestaciones económicas.

d) De financiación a través de impuestos generales, finalistas o cotizaciones.

e) Con cofinanciación de los beneficiarios o de carácter gratuito.

f) De gestión centralizada o descentralizada en las regiones y los municipios.

g) Alcance de la financiación de las Administraciones Públicas para la protección a la dependencia

A) De Cobertura universal en el Ámbito de LA PRotección pÚblica o en el marco regulador de la Seguridad Social

De los temas anteriores el que seguramente dio lugar a mayor debate fue el relacionado con el marco de protección, es decir, si la protección de la dependencia debería ser una prestación del Sistema de Seguridad Social o un sistema propio financiado por los presupuestos del Estado a través de impuestos.

A los efectos de decidir sobre el modelo que podía interesar a nuestro país se realiza un análisis detenido sobre la operatividad de los dos tipos de sistemas de atención a la dependencia dentro de nuestro modelo de protección social y de la organización del Estado. Para ello incluso se realizaron visitas programadas a Alemania, Holanda y Francia para contrastar las ventajas e inconvenientes de los dos modelos:

El modelo de protección universal para todos los ciudadanos y financiación mediante impuestos: Modelo de los países nórdicos y de Holanda.

- El modelo de protección en el marco del Sistema de la Seguridad Social, mediante prestaciones financiadas con cotizaciones: Modelo bis- 
markiano de los países centroeuropeos, vigente en Alemania, Austria y Luxemburgo.

- El modelo de protección universal atiende la dependencia como una extensión lógica de la concepción de que el Estado debe cubrir las necesidades básicas de los ciudadanos por razones de ciudadanía, de la misma forma que se atiende cualquier otra de las necesidades sanitarias o educativas.

En el modelo de Seguridad Social la protección social a la dependencia se garantiza en tanto constituye un riesgo social protegido, al igual que las pensiones, el seguro de desempleo o la protección a la familia, por lo tanto, financiado y gestionado en este marco.

En favor del sistema de protección universal, se argumentaba que al tratarse de una situación social y en algunos casos sociosanitaria, que afecta a la población en su condición de ciudadano, la mejor forma de abordarla era en términos similares a la cobertura de las necesidades de asistencia sanitaria, es decir, que las Administraciones Públicas habilitaran los servicios necesarios para dar cobertura a las situaciones de dependencia que se originen.

A favor de que la dependencia sea considerada como uno de los riesgos que deben ser protegidos mediante el seguro público, es decir, a través de una prestación del Sistema de la Seguridad Social, se argumentaba que la dependencia es una contingencia que puede producirse o no en las personas; solo una parte de ellas se verán afectadas a lo largo de su vida por dificultades o imposibilidad para realizar las actividades básicas de la vida diaria. Estaríamos, por tanto, ante un riesgo que puede ser cubierto por el Sistema de Seguridad Social. Los ciudadanos transfieren una parte pequeña de sus recursos mediante cotizaciones finalistas a un fondo común de Seguridad Social con el que se hará frente a las situaciones de dependencia que se produzcan, evitando con ello que cada ciudadano se vea obligado a ahorrar individualmente cantidades muy significativas, por si en algún momento de su vida aparece una deficiencia que de lugar a la dependencia.

La incorporación de la prestación de dependencia al Sistema de la Seguridad Social constituiría un paso más en el actual régimen de protección de la invalidez contributiva y no contributiva. La Ley General de Seguridad Social contempla en el artículo 137. 1.c) y 139.4 la Gran Invalidez. En este ultimo artículo se establece (redacción de la última reforma del 2007) que "Si el trabajador fuese calificado de gran inválido, tendrá derecho a una pensión vitalicia, incrementándose su cuantía con un complemento, destinado a que el inválido pueda remunerar a la persona que le atienda. El importe de dicho complemento será equivalente al resultado de sumar el 45 por ciento de la base mínima de cotización vigente en el momento del he- 
cho causante y el 30 por ciento de la última base de cotización del trabajador correspondiente a la contingencia de la que derive la situación de incapacidad permanente. En ningún caso el complemento señalado podrá tener un importe inferior al 45 por ciento de la pensión percibida, sin el complemento, por el trabajador".

El Libro Blanco señalaba: "No sería, por tanto, una novedad ampliar la acción protectora del Sistema, regulando esta nueva prestación, resolviendo, además, la falta de equidad, que actualmente se produce, de que el reconocimiento de la gran invalidez y del concurso de tercera persona sólo pueden serles reconocidas a las personas menores de sesenta y cinco años".

Este tema constituyó uno de los puntos clave de la negociación del Gobierno con los Interlocutores Sociales. En las negociaciones para la definición de un modelo de protección de la dependencia dentro de la agenda del diálogo social de éstos con el Gobierno de la Nación, se debatió como eje fundamental si esta nueva protección debería o no enmarcarse dentro del Sistema de la Seguridad Social o fuera de ella. La posición de los Sindicatos fue siempre hasta el final, en que ceden para alcanzar un acuerdo, que la protección de la dependencia debería ser una prestación más dentro de la Seguridad Social en el ámbito de las prestaciones no contributivas, aunque esto último no llegó nunca a debatirse por la posición contraria a que formara parte de ella, defendida por la representación empresarial. En algún momento estuvo encima de la mesa una propuesta de acercamiento, que consistía en darle a la protección de la dependencia la naturaleza de un servicio social de los contemplados por la Ley General de Seguridad Social para las personas mayores y con discapacidad, en su artículo 38, lo que supondría que la financiación sería, en todo caso, de los presupuestos del Estado a través de impuestos y no de Seguridad Social.

B) Protección de acceso abierto a toda persona con dependencia O LIMITADO A DETERMINADOS NIVELES DE SEVERIDAD

Los antecedentes de la atención a las personas con dependencia se encuentran en los servicios sanitarios. El origen de la dependencia es la enfermedad, por lo tanto, parecía lógico plantearse si el Sistema de la dependencia no debería ser una prolongación del Sistema sanitario y, en consecuencia, sometido a sus mismas reglas. Si la asistencia sanitaria cubre las necesidades de las personas independientemente del grado de severidad de la enfermedad, la pregunta obligada era si debía el nuevo sistema garantizar prestaciones a todas las personas con algún tipo de dependencia o si, por el contrario, 
debería limitar su alcance protector a los más dependientes, dejando con la protección actual menos garantista a los más moderados.

La protección alcanzada en los Países Nórdicos en los que la protección se extiende por igual no solo a los más severos, sino también a los moderados y ligeros, resultaba una meta difícil de alcanzar por su alto coste, partiendo de la escasa protección de nuestro país. El objetivo era proteger a las personas más gravemente afectadas con dificultades para realizar las actividades básicas de la vida diaria por razones no solo económicas sino también por la insuficiencia de servicios para atender a todos los ciudadanos.

Esta decisión conlleva, como en los países centroeuropeos, desarrollar un procedimiento de valoración, que pueda facilitar la elegibilidad de las personas que deben ser atendidas.

A través de la aplicación de un baremo se puede moderar el gasto. Resulta significativa la diferencia de costes entre quienes protegen a toda la población y los que solo lo hacen a los más severos, el 3 por ciento del PIB de los modelos nórdico frente al 1,5 de los centroeuropeo. No obstante, es preciso señalar que valorar a cada solicitante supone retrasar la prestación de los cuidados y la burocratización del proceso.

En la toma de decisión de aplicar baremo hubo un consenso generalizado, básicamente porque ha sido tradición en la valoración de la discapacidad y en el acceso a las pensiones de invalidez, contributivas y no contributivas. El baremo se elabora ex novo con la colaboración del Instituto del Envejecimiento de la Universidad Autónoma de Barcelona, adaptado a los requerimientos establecidos en el proyecto de ley.

La Ley en su artículo 27 2. 3. y 4 determina los aspectos que ha de valorar el baremo y determina como referente la Clasificación internacional del Funcionamiento, la Discapacidad y la Salud (CIF), adoptada por la Organización Mundial de la Salud.

“2. El grado y niveles de dependencia, a efectos de su valoración, se determinarán mediante la aplicación del baremo que se acuerde en el Consejo Territorial del Sistema para la Autonomía y Atención a la Dependencia para su posterior aprobación por el Gobierno mediante Real Decreto.

3. El baremo establecerá los criterios objetivos de valoración del grado de autonomía de la persona, de su capacidad para realizar las distintas actividades de la vida diaria, los intervalos de puntuación para cada uno de los grados y niveles de dependencia, y el protocolo con los procedimientos y técnicas a seguir para la valoración de las aptitudes observadas, en su caso.

4. El baremo valorará la capacidad de la persona para llevar a cabo por sí misma las actividades básicas de la vida diaria, así como la necesidad de apoyo y supervisión para su realización por personas con discapacidad intelectual o con enfermedad mental." 


\section{C) Prestaciones de servicios o prestaciones económicas}

Un tema fundamental era decidir sobre las modalidades de prestación que debían configurar el Sistema, si debía ser de servicios o de prestaciones económicas, así como el papel que debería representar el cuidado por familiares en el Sistema.

La posición mayoritaria de los interlocutores sociales, así como de las organizaciones de mayores y de personas con discapacidad es que debe ser un sistema de prestación de servicios, sin embargo existe también un sector de la sociedad y de los beneficiarios que prefiere las prestaciones económicas y con ellas decidir libremente sobre el servicio a adquirir, comúnmente conocido como cheque servicio.

De la lectura de la Ley parece desprenderse con nitidez que estamos ante un sistema de servicios. El artículo 14 establece la forma en que ha de entenderse y aplicarse el régimen de prestaciones de la Ley:

“2. Los servicios del Catálogo del artículo 15 tendrán carácter prioritario...

3. De no ser posible la atención mediante alguno de estos servicios, en los Convenios a que se refiere el artículo 10 se incorporará la prestación económica vinculada establecida en el artículo $17 \ldots$

4. El beneficiario podrá, excepcionalmente, recibir una prestación económica para ser atendido por cuidadores no profesionales, siempre que se den condiciones adecuadas de convivencia y de habitabilidad de la vivienda y así lo establezca su Programa Individual de Atención.”

Existen razones que avalan la garantía de servicios frente a las prestaciones económicas. Es la mejor forma de garantizar los cuidados que precisa la persona con dependencia y de que los servicios prestados generen empleo y actividad económica. Sin embargo, la ley debía prever un periodo transitorio en el que la garantía del derecho a la atención y al cuidado pudiera ofrecerse a través de cuidados en el entorno familiar. La insuficiencia de recursos para atender a todas las personas reconocidas hubiera dejado en papel mojado el derecho a la protección que predica la ley, si no se hubieran contemplado prestaciones económicas sustitutivas para cuando no hubiera servicios en el ámbito donde la persona tenga su residencia.

Por otra parte, la voluntad de las familias y de las personas con dependencia manifestada en múltiples encuestas es que mayoritariamente desean ser atendidos en su entorno familiar. La Ley debía contemplar esta opción, aunque le atribuye la naturaleza de excepcional. Sin embargo, la realidad de la gestión de la ley durante los dos años en vigor es que la prestación de cuidados en el entorno familiar constituye una prestación significativa del 
Sistema, como ha ocurrido en Alemania, en donde, dieciocho años después de la aplicación de su ley de protección de la dependencia, el cincuenta por ciento tiene este tipo de prestación, a pesar de que su cuantía es la mitad que la de carácter profesional.

\section{D) Financiación a través de impuestos generales, Finalistas o COTIZACIONES}

La financiación a través de impuestos es el resultado de la opción tomada de sacar fuera del Sistema de Seguridad Social la protección de la dependencia. Se plantean dudas sobre si la financiación del Sistema para la Autonomía y Atención a la Dependencia ha quedado bien resuelto en la Ley. Es cierto que el artículo 32. 1 de Ley dice que "la financiación del Sistema será la suficiente para garantizar el cumplimiento de las obligaciones que correspondan a las Administraciones Públicas competentes y se determinará anualmente en los correspondientes Presupuestos", sin embargo la cuestión es si la dotación presupuestaria anual, no solo de la Administración General del Estado sino también de las Comunidades Autónomas que son cofinanciadoras del Sistema es y será suficiente.

La suficiencia hubiera quedado mejor garantizada si la financiación se hubiera vinculado a un impuesto finalista variable en los tipos en función de las necesidades o mediante cotización. Cuando se elaboró el Libro Blanco en el barómetro del año 2004 se preguntó a los ciudadanos si estarían dispuestos a pagar más impuestos para la atención a la dependencia. Pues bien, el 70 por ciento dijeron que sí, siempre que se destinara a este fin.

\section{E) La distribución de la Financiación del Sistema entre las Administraciones Públicas}

La distribución de la financiación entre las Comunidades Autónomas y la Administración General del Estado constituye con seguridad uno de los aspectos menos pacíficos de la Ley. Para comprender las peculiaridades que incluye es preciso tener en cuenta que las competencias en materia de servicios sociales le están atribuidas a las Comunidades Autónomas, por lo tanto debe ser la Administración Autonómica quien asuma la mayor parte del coste del Sistema. Es cierto que al convertirse el Estado en promotor y regulador de este nuevo derecho debe ser corresponsable de su implantación y garantía.

La Ley regula la financiación del Sistema de forma insuficiente, puesto que, aunque atribuye que le corresponde al Estado la financiación del nivel 
mínimo de protección garantizado y que, asimismo, participará en la financiación del nivel acordado, no llega a determinarse en qué porcentaje del coste total del Sistema habrá de participar el Estado y en qué porcentaje la Comunidad Autónoma. Y no debemos olvidar que lo que no ponga una Administración lo habrá de poner la otra.

En estos dos primeros años de puesta en marcha de la Ley se ha planteado como cuestión de debate si la financiación de la Ley debe incluir a las personas ya atendidas a la entrada en vigor con servicios de los establecidos en el catálogo aprobado (plazas residenciales y de centros de día). La memoria económica de la Ley calculaba la financiación de la Ley, estimando el coste que representaría la atención a la totalidad de los beneficiarios previstos, cuando la Ley estuviera plenamente implantada. Ahora bien, como quiera que una parte de los beneficiarios potenciales estaban ya siendo atendidos en los centros y servicios existentes gestionados por las Comunidades Autónomas, para calcular el coste que representaría poner en marcha la Ley era necesario descontar las cantidades con las que se financiaban los centros y servicios en funcionamiento para personas con dependencia a la entrada en vigor de la ley. El resultado final es que la memoria de la Ley, como era lógico, solo recoge como gasto a financiar el gasto nuevo derivado de la incorporación de los nuevos beneficiarios y no de los atendidos en plazas existentes y ya financiadas.

El texto articulado no incorpora ninguna disposición sobre la financiación de los servicios ya financiados, por lo que entienden las Comunidades Autónomas que la financiación del Estado debe ir destinada no solo a los nuevos beneficiarios, sino también a los ya atendidos. Esta interpretación, que puede ser válida en la aplicación individualizada de la Ley a cada beneficiario, — de hecho la Administración General del Estado abona el mínimo garantizado por cada beneficiario reconocido como dependiente independientemente de antiguo o nuevo-, no lo es cuando se analiza la aportación del Estado y de las Comunidades Autónomas a la financiación del Sistema y la suficiencia o insuficiencia de la misma. La financiación del Estado para la puesta en marcha de la ley y la extensión de la protección a las personas reconocidas como dependientes no debe ir destinada a refinanciar plazas de servicios ya financiadas en los presupuestos.

\section{F) Debe HABER COFINANCIACIÓN DE LOS BENEFICIARIOS O LAS PRESTACIONES DEBEN SER DE CARÁCTER GRATUITO}

Otro de los grandes temas que fue muy debatido durante la tramitación del proyecto de ley y después hasta la aprobación en fechas recientes del 
Acuerdo de determinación de la capacidad económica y participación de los beneficiarios en el coste de los servicios, es el de la cofinanciación, popularmente conocido como "copago". En el capítulo dedicado al desarrollo de la ley de este monográfico se hace un detallado análisis del Acuerdo aprobado por el Consejo Territorial.

Era necesario decidir si el sistema de la dependencia incluiría copago o si, como en las prestaciones sanitarias o educativas, debería ser gratuito. He de decir que, salvo el Grupo parlamentario de Izquierda Unida y el CERMI que defendieron la gratuidad total, la mayor parte de las organizaciones y grupos entendieron que era necesaria la participación económica de los beneficiarios.

El "copago" ha formado parte de la estructura de financiación de los servicios sociales en nuestro país, salvo excepciones en el sector de la discapacidad en alguna Comunidad Autónoma. Por otra parte, es general la participación económica del beneficiario en todos los países que han establecido este régimen de protección, incluso en los países nórdicos.

La cuestión que se ha planteado durante todo este tiempo no ha sido, por tanto, si debería haber copago o no, sino más bien el alcance del mismo, hasta dónde debería llegar en relación con los costes. La memoria económica de la ley calculaba la aportación del beneficiario en un 33 por ciento, sin embargo las disposiciones de la Ley sobre esta materia no fijaban criterios precisos que permitieran alcanzar ese nivel de cofinanciación. El artículo 33 sobre participación de los beneficiarios en el coste de las prestaciones remite a un posterior Acuerdo del Consejo la fijación de criterios, señalando que deberá tenerse en cuenta el tipo y coste del servicio y la capacidad económica personal, así como la garantía de que ningún ciudadano quedará fuera de la cobertura del Sistema por no disponer de recursos económicos.

No resulta fácil saber con el Acuerdo aprobado cual será la participación del beneficiario en los costes en términos globales. El Acuerdo contempla intervalos que han de ajustar las Comunidades Autónomas, fijando tablas concretas de cofinanciación. No obstante, según cálculos efectuados por algunas Comunidades Autónoma, con los beneficiarios actuales la participación no superará el 25 por ciento en residencias y el 15 por ciento en otros servicios.

\section{G) Gestión centralizada o descentralizada en las CC.AA. y los MUNICIPIOS}

Sobre la gestión del Sistema no había duda de que les corresponde a las Comunidades Autónomas, una vez descartado su enmarque dentro del Sistema de Seguridad Social. La cuestión era y es cuál debe ser el papel que han de desempeñar los municipios en la aplicación del Sistema. 
La Ley no podía llegar más allá de lo que dispone. Dedica los artículos 11 y 12 a definir la participación de las Comunidades Autónomas y las Corporaciones Locales, con una remisión genérica a la normativa de sus respectivas Comunidades Autónomas y dentro de las competencias que la legislación vigente les atribuye. La participación de los municipios será en definitiva la que en cada Comunidad Autónoma se determine y acuerde con ellos.

La Federación Española de Municipios y Provincias pretendió que la ley dejara más claro el papel de las entidades locales en la aplicación de la Ley. No fue posible sin que el Estado entrara en conflicto con las competencias exclusivas de las Comunidades Autónomas en la gestión de los servicios sociales.

El resultado es que coexisten una variedad de modelos de participación de los municipios en la gestión, que entiendo se irán reconduciendo con el tiempo, desde Comunidades Autónomas en las que la gestión se encuentra totalmente centralizada y los municipios no participan en ninguno de los procesos, hasta Comunidades en donde los municipios participan en la valoración de la situación de dependencia, elaboración del PIA y la prestación de los servicios de teleasistencia y ayuda a domicilio, pasando por aquellas en que la participación es la de apoyo en la elaboración del PIA o en la prestación de alguno de estos servicios.

La clave de la diversidad, considero que no está solo en la voluntad política de las Comunidades Autónomas de delegar parcelas de gestión en las entidades locales, sino en la insuficiencia financiera de estas Entidades y en la dependencia económica de las Comunidades Autónomas para la atención de los servicios sociales que gestionan.

\section{NEGOCIACIÓN CON LOS INTERLOCUTORES SOCIALES}

Durante el año 2005 el Gobierno desarrolla con los Interlocutores Sociales un proceso de negociación, basado en el Acuerdo sobre Competitividad, Empleo Estable y Cohesión Social, firmado el 8 de julio del 2004, con el fin de adaptar "nuestra protección social a las nuevas realidades, que configuran nuevas necesidades, siendo prioritaria la atención a las situaciones de dependencia, uno de los principales problemas tanto de los jubilados y discapacitados como de sus familias".

Ambas partes coincidían en que la atención de las necesidades de las personas en situación de dependencia, personas mayores y personas con discapacidad, se ha convertido, sin duda, en un reto para las políticas públicas ante las dificultades y costes que la dependencia supone para cientos de miles de familias.

Asimismo, coinciden en que la atención a quienes se encuentran en situación de dependencia es una exigencia para avanzar en la sociedad del 
bienestar y en la construcción de una sociedad más justa y más digna. Este objetivo se inscribe en el modelo de Estado social que consagra la Constitución Española y en la que los Poderes Públicos han de garantizar dicha atención, instrumentando las políticas públicas adecuadas. Por lo que el Gobierno y las organizaciones sindicales y empresariales están de acuerdo en el desarrollo de un nuevo sistema de protección a las personas en situación de dependencia y sus familias.

Para el Gobierno y las organizaciones sindicales y empresariales la implantación de un sistema de promoción de la autonomía personal y de atención a la dependencia es un avance fundamental en la protección social que configura el elemento central del cuarto pilar del estado del bienestar, estableciendo un nuevo derecho de ciudadanía, de carácter subjetivo.

Consideran también que la promoción de la autonomía personal y atención a las personas en situación de dependencia ha de ser compartida por todas las Administraciones, en el ejercicio de sus competencias. La intervención de la Administración General del Estado está legitimada por el mandato constitucional de que todos los españoles han de tener los mismos derechos y obligaciones en cualquier parte del territorio español (art. 139.1 CE), así como por la competencia estatal para la regulación de las condiciones básicas que garanticen la igualdad de todos los españoles en el ejercicio de los derechos y en el cumplimiento de los deberes constitucionales (art. 149.1.1 CE).

En consonancia con lo expuesto, el Gobierno y las organizaciones sindicales y empresariales acuerdan impulsar un Sistema Nacional de Dependencia mediante una ley, que promueva la autonomía personal y garantice el derecho a ser atendido en situaciones de dependencia, y en la que se establecerán las condiciones básicas y los requisitos de acceso a los servicios y prestaciones en condiciones de equidad en todo el territorio del Estado. El Sistema Nacional de la Dependencia se configura como una nueva modalidad de protección social que amplía y complementa la acción protectora de la Seguridad Social y del Estado.

Se destacan algunos de los acuerdos significativos del documento firmado por la representación del Gobierno, de las organizaciones empresariales COE y CEPYME y de las organizaciones sindicales UGT y CC.OO. el día 21 de diciembre:

\section{a) Los principios del Sistema Nacional de Dependencia}

- El carácter universal y público de las prestaciones.

- El acceso a las prestaciones de dependencia en condiciones de igualdad y no discriminación

- La equidad en la distribución territorial de los recursos 
- La promoción de las condiciones precisas para que las personas en situación de dependencia puedan llevar una vida con el mayor grado de autonomía posible.

- La permanencia de las personas en situación de dependencia, siempre que sea posible, en el entorno en el que desarrollan su vida.

- La colaboración de los servicios sociales y sanitarios, públicos y privados, con y sin ánimo de lucro, en la prestación de los servicios a los usuarios del Sistema Nacional de Dependencia.

- La coordinación y colaboración interadministrativa.

b) El derecho a los servicios y prestaciones de promoción de la autonomía personal y atención a la dependencia de los españoles con una edad superior a 3 años que se encuentren en situación de dependencia en alguno de los grados establecidos.

c) La valoración de la dependencia con tres grados:

a) Dependencia moderada: Cuando la persona necesita ayuda para realizar varias actividades básicas de la vida diaria, al menos una vez al día.

b) Dependencia severa: Cuando la persona necesita ayuda para realizar varias actividades básicas de la vida diaria dos o tres veces al día, pero no requiere la presencia permanente de un cuidador.

c) Gran dependencia: Cuando la persona necesita ayuda para realizar varias actividades básicas de la vida diaria varias veces al día y, por su pérdida total de autonomía mental o física, necesita la presencia indispensable y continua de otra persona.

Cada uno de los grados de dependencia se clasificarán en dos niveles, en función de la autonomía de la persona y de la intensidad del cuidado que requiere.

El grado y niveles de dependencia se determinará mediante un sistema público de valoración a través de un baremo común en todo el territorio del Estado, que determine el grado de autonomía de las personas y su capacidad para realizar las distintas actividades de la vida diaria.

\section{d) La configuración del Sistema Nacional de Dependencia}

El Sistema Nacional de Dependencia se configura como un sistema integrado de prestaciones y servicios de prevención, atención y protección a las personas en situación de dependencia a través de una red que integre de forma coordinada centros y servicios públicos y privados concertados debi- 
damente acreditados, con la cooperación de todas las Administraciones Públicas en el ejercicio de sus competencias.

El catálogo de servicios responderá a dos modalidades:

1. Los Servicios para la promoción de la autonomía personal que comprende la prevención, la teleasistencia, las ayudas técnicas para la autonomía personal, las ayudas para la adaptación y accesibilidad del hogar y la asistencia personalizada.

2. Los Servicios de atención y cuidado comprenden: el Servicio de ayuda a domicilio (atención de las necesidades del hogar, cuidados personales), el Servicio de atención en Centros de Día y de Noche (centros para mayores, de atención a menores de 65 años y de atención especializada), el Servicio de atención en centro residencial (residencias de personas mayores dependientes, centros de atención a personas con discapacidad)

La prestación de servicios a través de la oferta del Sistema de titularidad pública o concertada tendrá carácter prioritario. De no ser posible, se procederá al reconocimiento de una prestación económica vinculada, suficiente y destinada a la provisión de la atención a través de servicios privados. Se establecerán mecanismos de control para que la utilización de estas prestaciones se destinen al cumplimiento de su finalidad.

Sin perjuicio de lo anterior, siempre que se den las circunstancias que garanticen unos cuidados adecuados, según el programa individual de atención, la persona en situación de dependencia podrá ser atendida en su ámbito familiar, recibiendo una compensación económica por ello, variable en función del grado de dependencia.

Esta prestación en el ámbito familiar conllevará programas de información, formación y respiro para los cuidadores, encargados de la atención de las personas en situación de dependencia.

Se regulará la incorporación de los cuidadores familiares al Régimen de la Seguridad Social que se determine. La cotización empresarial será con cargo al Sistema Nacional de Dependencia.

Asimismo, el sistema ofrecerá protección jurídica a las personas en situación de dependencia ante situaciones en las que se impida o dificulte el ejercicio de sus derechos, mediante la regulación de un régimen específico de infracciones y sanciones.

\section{e) La aplicación progresiva del sistema}

El periodo de implantación del sistema se extenderá desde el 2007 al 2015, fecha en la que estará plenamente implantado. Transcurridos los 
primeros cinco años se realizará una evaluación de los resultados de la misma.

El reconocimiento de las prestaciones de dependencia se realizará con un calendario progresivo, iniciándose por las personas más afectadas. El primer año serán reconocidas las prestaciones de quienes sean valorados en el Grado III, niveles 1 y 2.

\section{f) Financiación del sistema}

Los servicios y prestaciones del Sistema Nacional de Dependencia serán financiados por las Administraciones Públicas y se complementarán con aportaciones de los beneficiarios. La financiación del sistema será estable, suficiente y sostenible, asegurando la corresponsabilidad de todos los agentes implicados.

La financiación de las Administraciones Públicas se determina en el Consejo de Política Fiscal y Financiera. Durante el período de implantación progresiva del sistema, la Administración General del Estado establecerá anualmente en sus Presupuestos los créditos determinados para la atención a las personas en situación de dependencia. Su aportación será del $50 \%$ de la financiación pública del coste nuevo que represente la implantación del sistema y tendrá carácter finalista con objetivos concretos y cuantificables, e irá destinada prioritariamente a incrementar la red de servicios públicos, a ampliar el número de personas atendidas mediante concierto y a mejorar la intensidad de los servicios; asimismo, esta financiación posibilitará el acceso a servicios privados, cuando no sea posible garantizar un servicio de titularidad pública o concertada, y compensará económicamente a quienes sean atendidos en el medio familiar.

El sistema atenderá de forma equitativa a todos los ciudadanos en situación de dependencia. Ningún ciudadano en situación de dependencia quedará sin atender por falta de recursos. Los beneficiarios contribuirán económicamente a la financiación de los servicios de forma progresiva en función de su capacidad económica personal, teniendo en cuenta para ello el tipo de servicio que se presta y el coste del mismo, garantizando en los servicios de atención residencial un mínimo para gastos personales. La prestación económica vinculada al servicio y la destinada a cuidados familiares se determinarán, asimismo, en función de la capacidad económica global de la persona dependiente.

Cuando esté plenamente implantado, se estima que el coste anual del sistema se situará en torno a un punto del PIB, teniendo en cuenta el número de beneficiarios determinado en el Libro Blanco de la Dependencia y la protección establecida en el catálogo. 


\section{g) Calidad del sistema}

El sistema fomentará la calidad en la prestación de los servicios de atención a la dependencia con el fin de asegurar, de forma eficiente, los derechos de las personas en situación de dependencia; para ello, se incorporarán las condiciones comunes de acreditación de los servicios y de los centros, los estándares de calidad de los servicios y calidad del empleo, los indicadores, las guías de buenas prácticas, las cartas de servicios, los sistemas de evaluación, investigación, desarrollo e innovación. Además, se establecerá un sistema común de información.

\section{h) Formación y cualificación de profesionales y cuidadores}

Se determinarán las cualificaciones profesionales más idóneas para el ejercicio de las funciones que se correspondan con el catálogo de servicios y se promoverán los planes de formación que sean necesarios para la implantación de los títulos de formación profesional y certificados de profesionalidad correspondientes a estos servicios, todo ello en el marco del Sistema Nacional de las Cualificaciones.

Para el desarrollo e impulso de planes de formación se creará un Centro de Referencia Nacional de Servicios Sociales y Dependencia, dependiente del Ministerio de Trabajo y Asuntos Sociales.

\section{i) Comité Consultivo}

Se creará un Comité Consultivo a través del cual se hará efectiva, de manera permanente, la participación institucional de las organizaciones sindicales y empresariales más representativas. El mismo tendrá carácter tripartito (integrado por Administraciones Públicas, organizaciones sindicales y organizaciones empresariales) y paritario en la adopción de acuerdos.

\section{j) Seguimiento del Acuerdo}

El Gobierno y los interlocutores sociales se comprometen a continuar el Diálogo Social en el desarrollo normativo del Sistema Nacional de Dependencia. Para el análisis y seguimiento de este acuerdo y, en general, de la evolución del Sistema Nacional de Dependencia, se acuerda dar continuidad a la Mesa de Dependencia, actualmente constituida e integrada por las partes firmantes de este acuerdo. 
La valoración que se puede hacer de este Acuerdo es que ha sido fundamental para la configuración del modelo de protección social de la dependencia, sin el, seguramente el Proyecto de Ley no hubiera tenido la acogida que tuvo en el Parlamento entre los Grupos Parlamentarios y entre las organizaciones de mayores y personas con discapacidad y en general en la sociedad, a quien los interlocutores sociales se encargaron de comunicar las ventajas del proyecto.

En este Acuerdo están los elementos sustantivos de la Ley, la naturaleza de derecho subjetivo de la protección, los principios en que se fundamenta el Sistema, el catálogo de servicios y prestaciones económicas, la prioridad de los servicios, el carácter excepcional de la prestación de cuidados en el entorno familiar, la financiación y la aplicación progresiva.

\section{APROBACIÓN DEL ANTEPROYECTO Y PROYECTO POR EL GOBIERNO: COMISIÓN DE PRESIDENCIA}

La aprobación del Anteproyecto y del Proyecto de Ley por el Gobierno no fue tarea fácil, hubo que poner de acuerdo a los distintos Ministerios que por razón de competencias estaban relacionados con el proyecto: Trabajo y Asuntos Sociales, hoy Educación, Política Social y Deporte, promotor de la Ley, Sanidad, Administraciones Públicas, Economía y Hacienda y Presidencia del Gobierno. De ello se encargó una Comisión, constituida ad hoccon representantes del mayor nivel de los Departamentos y el apoyo técnico correspondiente, bajo la presidencia del Director del Gabinete del Presidente del Gobierno.

Los textos del Anteproyecto y Proyecto de Ley salieron de esta Comisión enriquecidos principalmente desde punto de vista de su fundamentación constitucional, con una mayor coherencia técnica y un Capitulo V destinado a la financiación del Sistema acordado con el Ministerio de Economía y Hacienda.

Del trabajo de esta Comisión es preciso destacar el anclaje constitucional del Proyecto dentro del respeto a las competencias del Estado y de las Comunidades Autónomas y del principio de lealda institucional. El fundamento constitucional, que se recoge en la disposición final octava: "Esta Ley se dicta al amparo de la competencia exclusiva del Estado para regular las condiciones básicas que garanticen la igualdad de todos los españoles en el ejercicio de los derechos y el cumplimiento de los deberes constitucionales, conforme al artículo 149.1.1 de la Constitución", tiene su reflejo directo en el establecimiento de los tres niveles de protección que se establecen en el artículo 7 y específicamente en la regulación del nivel mínimo de protección 
garantizado en el artículo 9. El nivel mínimo se establece como "condición básica de garantía del derecho a la promoción de la autonomía personal y atención a la situación de dependencia". El Estado no solo establece y regula este nivel mínimo, sino que lo financia íntegramente: "La financiación pública de este nivel de protección correrá a cuenta de la Administración General del Estado que fijará anualmente los recursos económicos en la Ley de Presupuestos Generales del Estado de acuerdo con los criterios establecidos en el artículo 32".

Por otra parte, la Administración General del Estado colabora en la financiación del nivel acordado: Artículo 10 1. "En el seno del Consejo Territorial del Sistema para la Autonomía y Atención a la Dependencia, la Administración General del Estado y las Comunidades Autónomas acordarán el marco de cooperación interadministrativa que se desarrollará mediante los correspondientes Convenios entre la Administración General del Estado y cada una de las Comunidades Autónomas.

2. A través de los Convenios a los que se refiere el apartado anterior, la Administración General del Estado y las Comunidades Autónomas acordarán los objetivos, medios y recursos para la aplicación de los servicios y prestaciones recogidos en el Capítulo II del presente Título, incrementando el nivel mínimo de protección fijado por el Estado de acuerdo con el artículo 9.”

Esta forma de regular las condiciones básicas, que se incorporan al texto del proyecto en base a las reflexiones que tienen lugar en el seno de la Comisión, fueron valoradas positivamente en el informe del Consejo de Estado al Anteproyecto de Ley, aunque recomendó una mayor precisión en la conceptualización del los tres niveles; mejora que fue incorporada al Proyecto de Ley aprobado por el Consejo de Ministros.

Otro de los aspectos que fue incorporado al Proyecto en el trabajo de la Comisión fue el carácter otorgado al Consejo Territorial como órgano fundamental y decisivo en la configuración y desarrollo del Sistema. Dice la exposición de motivos que "la propia naturaleza del objeto de esta Ley requiere un compromiso y una actuación conjunta de todos los poderes e instituciones públicas, por lo que la coordinación y cooperación con las Comunidades Autónomas es un elemento fundamental. Por ello, la ley establece una serie de mecanismos de cooperación entre la Administración General del Estado y las Comunidades Autónomas, entre los que destaca la creación del Consejo Territorial del Sistema para la Autonomía y Atención a la Dependencia. En su seno deben desarrollarse, a través del acuerdo entre las administraciones, las funciones de acordar el marco de cooperación interadministrativa, la intensidad de los servicios del catálogo, las condiciones y cuantía de las prestaciones económicas, los criterios de participación de los 
beneficiarios en el coste de los servicios o el baremo para el reconocimiento de la situación de dependencia, aspectos que deben permitir el posterior despliegue del Sistema a través de los correspondientes convenios con las Comunidades Autónomas".

En relación con el tratamiento de los aspectos sanitarios y sociosanitarios en la ley, la Comisión a instancias del Ministerio de Sanidad consideró que esta ley debía regular los aspectos sociales de la atención a la dependencia, entendiendo que los sanitarios y sociosanitarios estaban ya regulados en la Ley de sanidad y en la Ley de cohesión y calidad del Sistema de Salud. Así pues el artículo 15 de la Ley determina los servicios sociales que constituyen el catálogo de servicios, añadiendo que: "Los servicios establecidos en el apartado 1 se regulan sin perjuicio de lo previsto en el artículo 14 de la Ley 16/2003, de 28 de mayo, de Cohesión y Calidad del Sistema Nacional de Salud", referido a las prestaciones sociosanitarias. Por otra parte, deja en el ámbito de las Comunidades Autónomas la coordinación de los aspectos sociales y sanitarios de la atención, considerando que estamos ante aspectos organizativos de su exclusiva competencia.

Por lo que se refiere a la financiación, se incorpora a propuesta del Ministerio proponente dos principios fundamentales que le dan garantía al Sistema, me refiero al de suficiencia en el texto articulado, artículo 32: "La financiación del Sistema será la suficiente para garantizar el cumplimiento de las obligaciones que correspondan a las Administraciones Públicas competentes y se determinará anualmente en los correspondientes Presupuestos" y al de sostenibilidad en la exposición de motivos: "La financiación vendrá determinada por el número de personas en situación de dependencia y de los servicios y prestaciones previstos en esta Ley, por lo que la misma será estable, suficiente, sostenida en el tiempo y garantizada mediante la corresponsabilidad de las Administraciones Públicas."

En este órgano se decide también el carácter transitorio de la financiación a través de convenios de la Administración General del Estado con las Comunidades Autónomas, limitando su duración al periodo de implantación: "Durante el período comprendido entre el 1 de enero de 2007 y el 31 de diciembre de 2015, y para favorecer la implantación progresiva del Sistema, la Administración General del Estado establecerá anualmente en sus Presupuestos créditos para la celebración de los convenios con las administraciones de las Comunidades Autónomas de acuerdo con el artículo 10 de esta Ley". Dudo que esta aportación financiera del la Administración General del Estado pueda interrumpirse sin un grave quebranto de la sostenibilidad del Sistema. Otra cuestión sería valorar la conveniencia de unificar los fondos destinados al nivel acordado con los establecidos para la financiación del mínimo garantizado. 


\section{LA PARTICIPACIÓN DE LAS PERSONAS CON DISCAPACIDAD Y DE LOS MAYORES}

Quisiera destacar aquí la gran labor que en defensa de los intereses legítimos de las personas con discapacidad realiza el CERMI, entre la aprobación del Anteproyecto y el Proyecto de Ley por el Gobierno y durante la tramitación parlamentaria del mismo. En la primera fase y tras un análisis detallado del anteproyecto consiguió la apertura de un proceso de negociación con el IMSERSO para analizar cada una de las propuestas presentadas, bastantes de ellas fueron incorporadas. Ya en fase de tramitación parlamentaria, muchas de las enmiendas presentadas por los Grupos Parlamentarios son el fruto del trabajo realizado por el CERMI, tanto de los Grupos que desde el inicio apoyaban la Ley como de los que no lo hacían. El alcance de este trabajo no me permite hacer un análisis detenido de los apartados de la Ley en los que se han incorporado posicionamientos de la discapacidad, no obstante, quisiera destacar su participación en la configuración de los artículos 3 y 4 dedicados a los principios y derechos, en la fijación de los conceptos de promoción de la autonomía personal y necesidades de apoyo, en la incorporación al catálogo del servicio de "promoción de la autonomía personal", así como en la inclusión de la necesidad de apoyo en la determinación de los grados de dependencia.

También quisiera referirme al excelente trabajo realizado por el Consejo Estatal de Mayores en representación de las organizaciones de mayores de todo el país. Desde la elaboración del Libro Blanco se constituyó un grupo de trabajo de dependencia dentro del Consejo, que ha venido trabajando ininterrumpidamente, analizando los distintos proyectos elaborados, realizando interesantes propuestas durante todo el proceso, especialmente las relacionadas con el catálogo de prestaciones y la participación de los beneficiarios en la financiación del Sistema, especialmente de las personas con menores recursos. El IMSERSO durante la elaboración del Anteproyecto ha sometido al Consejo Estatal sus propuestas, contrastando de esta forma la viabilidad de las mismas en las sucesivas reuniones de este órgano.

\section{LA TRAMITACIÓN PARLAMENTARIA DE LA LEY}

Durante la tramitación parlamentaria, el Gobierno, a través del Ministerio proponente, y el Grupo Parlamentario socialista negociaron con los Grupos Parlamentarios el apoyo al texto, que dio lugar a la aprobación de la Ley por todos los Grupos Parlamentarios, con la excepción del Grupo Catalán y el Grupo Vasco. 
El Ministerio de Trabajo y Asuntos Sociales suscribió el 19 de abril de 2006 un Acuerdo con los Grupos Parlamentarios de Izquierda Unida Iniciativa per Catalunya Verds y con Esquerra Republicana de Catalunya, por el que ambos grupos asumían la competencia del Estado para legislar en esta materia y se comprometían a apoyar la Ley siempre que se respetaran aquellos aspectos que formaban parte de la declaración conjunta:

- La garantía de un derecho subjetivo a la promoción de la autonomía personal y atención a las personas en situación de dependencia, de carácter universal y exigible administrativa y jurisdiccionalmente.

- Un modelo de financiación estable, suficiente y sostenido

- El acceso a los servicios en condiciones de igualdad

Durante la tramitación parlamentaria también se suscribió Acuerdo escrito de fecha 5 de junio de 2006 con el Boque Nacionalista Gallego, por el que se apoyaba la aprobación de la Ley, al mismo tiempo que se reconocían las peculiaridades que concurrían en Galicia respecto de la población dependiente y la insuficiencia de recursos, manifestando la voluntad desde el $\mathrm{Mi}$ nisterio de Trabajo de apoyar desde ambos Gobiernos la mejora de los servicios en Galicia.

Terminado el periodo de enmiendas se abrieron conversaciones con todos los Grupos, también con el Grupo Popular, buscando elementos comunes que no desvirtuaran el Proyecto de Ley. Entre las enmiendas aceptadas a este Grupo, se pueden citar algunas en la exposición de motivos para dejar constancia de actuaciones desarrollas durante el Gobierno del PP en materia de atención a personas mayores y con discapacidad, la incorporación de la prioridad en la atención a las personas con gran dependencia entre los principios de la Ley (constatación de la prioridad establecida en la disposición final primera en la que se fija el calendario de entrada en vigor por grados y niveles), y la determinación de los criterios objetivos del artículo 32 para el reparto de los créditos del nivel acordado.

El resultado de estas conversaciones fue al apoyo de Grupo Popular, y la negativa al mismo del Catalán y Vasco, fundamentado en la falta de competencias del Estado para regular, señalando que no contaba con título competencial específico que le habilitara para ello. De este asunto se trata de forma pormenorizada en alguno de los artículos de este monográfico.

De la tramitación del Proyecto de ley en el Congreso destacaría como elementos más significativos los siguientes:

a) La concreción en el artículo primero del carácter de derecho subjetivo de ciudadanía, "La presente Ley tiene por objeto regular las condi- 
ciones básicas que garanticen la igualdad en el ejercicio del derecho subjetivo de ciudadanía a la promoción de la autonomía personal y atención a las personas en situación de dependencia, en los términos establecidos en las leyes"

b) La definición del concepto de asistencia personal, que no estaba incluido, como el servicio prestado por un asistente personal que realiza o colabora en tareas de la vida cotidiana de una persona en situación de dependencia, de cara a fomentar su vida independiente, promoviendo y potenciando su autonomía personal.

c) La inclusión de forma expresa de los menores de tres años entre los titulares del derecho "para los menores de 3 años se estará a lo dispuesto en la Disposición Adicional decimotercera”.

d) La presencia mayoritaria de los representantes de las Comunidades Autónomas en el Consejo Territorial, así como la participación en este órgano de las Corporaciones Locales.

e) El carácter transitorio de la prestación económica vinculada "Hasta que la red de servicios esté totalmente implantada, las personas en situación de dependencia que no puedan acceder a los servicios por aplicación del régimen de prioridad señalado, tendrán derecho a la prestación económica prevista en el artículo 17 de esta Ley".

f) La obligación de que el Consejo Territorial acuerde "criterios comunes de composición y actuación de los órganos de valoración de las Comunidades Autónomas que, en todo caso, tendrán carácter público”.

g) La incorporación de que el baremo valorará no solo la capacidad de la persona para llevar a cabo por sí misma las actividades básicas de la vida diaria, sino también "la necesidad de apoyo y supervisión para su realización por personas con discapacidad intelectual o con enfermedad mental”.

h) La valoración se realizará teniendo en cuenta los correspondientes informes sobre la salud de la persona y sobre el entorno en el que viva.

i) Los servicios de valoración de la situación de dependencia, la prescripción de servicios y prestaciones y la gestión de las prestaciones económicas previstas en la presente Ley, se efectuarán directamente por las Administraciones Públicas, no pudiendo ser objeto de delegación, contratación o concierto con entidades privadas

j) La obligación de que el reparto de los créditos la Administración General del Estado se llevará a cabo, teniendo en cuenta "la población dependiente, la dispersión geográfica, la insularidad, emigrantes retornados y otros factores; y podrán ser revisados por las partes".

k) Para fijar la participación del beneficiario, se tendrá en cuenta la distinción entre servicios asistenciales y de manutención y hoteleros. 
Por lo que se refiere a la tramitación en el Senado y su aprobación en segunda vuelta en el Congreso es preciso destacar las siguientes modificaciones:

a) La incorporación al concepto de dependencia de la situación de larga duración en que se encuentran precisan de la atención de otra u otras personas o ayudas importantes para realizar actividades básicas de la vida diaria "o, en el caso de personas con discapacidad intelectual o mental, de otros apoyos para su autonomía personal".

b) Se definen las Necesidades de apoyo para la autonomía personal como "las que requieren las personas con discapacidad intelectual o mental para hacer efectivo un grado satisfactorio de autonomía personal en la comunidad".

c) Para ser titular de derechos se requiere ser reconocida como persona en situación de dependencia o "necesitar apoyos para su autonomía personal" en alguno de los grados establecidos.

d) En el catálogo de servicios se incorporan los servicios de Promoción de la Autonomía Personal.

e) Se modifican en el Artículo 26 los grados de dependencia, incorporando en cada uno de ellos las necesidades de apoyo que necesita:

- En el Grado I. Dependencia moderada: cuando la persona necesita ayuda para realizar varias actividades básicas de la vida diaria, al menos una vez al día "o tiene necesidades de apoyo intermitente o limitado para su autonomía personal”.

- En el Grado II. Dependencia severa: cuando la persona necesita ayuda para realizar varias actividades básicas de la vida diaria dos o tres veces al día, pero no quiere el apoyo permanente de un cuidador "o tiene necesidades de apoyo extenso para su autonomía personal".

- En el Grado III. Gran dependencia: cuando la persona necesita ayuda para realizar varias actividades básicas de la vida diaria varias veces al día y, por su pérdida total de autonomía física, mental, intelectual o sensorial necesita el apoyo indispensable y continuo de otra persona "o tiene necesidades de apoyo generalizado para su autonomía personal”.

f) El baremo valorará, además de la capacidad de la persona para llevar a cabo por sí misma las actividades básicas de la vida diaria, "la necesidad de apoyo para la autonomía personal en la comunidad de las personas con discapacidad intelectual o enfermedad mental".

En conclusión, podemos afirmar que la Ley durante la tramitación parlamentaria amplía significativamente su ámbito de protección, tanto en lo que se refiere al número de beneficiarios como a los servicios del catálogo. 


\section{Contexto actual de las políticas relativas a la promoción de la autonomía y la atención a la dependencia}


